\title{
Low serum potassium levels and risk of type 2 diabetes: the Toranomon Hospital Health Management Center Study 1 (TOPICS 1)
}

\author{
Y. Heianza • S. Hara • Y. Arase • K. Saito • K. Totsuka • \\ H. Tsuji • S. Kodama • S. D. Hsieh • N. Yamada • \\ K. Kosaka • H. Sone
}

Received: 31 August 2010 /Accepted: 3 December 2010 /Published online: 7 January 2011

(C) Springer-Verlag 2010

\begin{abstract}
Aims/hypothesis Evidence has suggested that low serum potassium concentrations decrease insulin secretion, leading to glucose intolerance, and that hypokalaemia induced by diuretics increases the risk for diabetes in hypertensive individuals. However, no prospective study has investigated the association between serum potassium and the development of type 2 diabetes in a healthy cohort comprised of Asian individuals not being administered antihypertensive medications. This study aimed to investigate whether low serum potassium is associated with increased risk of type 2 diabetes in apparently healthy Japanese men.

Methods We followed 4,409 Japanese men with no history of diabetes, use of antihypertensives, renal dysfunction or liver dysfunction (mean $\pm \mathrm{SD}$ age, $48.4 \pm 8.4$ years). Cox proportional hazards regression was used to estimate HRs for incident diabetes (fasting plasma glucose level $\geq 7.0 \mathrm{mmol} / \mathrm{l}$, $\mathrm{HbA}_{1 \mathrm{c}} \geq 6.5 \%$ or self-reported) including serum potassium concentration as either a categorical or a continuous variable.
\end{abstract}

Y. Heianza $\cdot$ K. Saito $\cdot$ K. Totsuka $\cdot$ S. Kodama $\cdot$ N. Yamada $\cdot$

H. Sone

Department of Internal Medicine,

University of Tsukuba Institute of Clinical Medicine,

Ibaraki, Japan

Y. Heianza $\cdot$ S. Hara $\cdot$ Y. Arase $\cdot$ K. Saito $\cdot$ K. Totsuka $\cdot$ H. Tsuji $\cdot$

S. Kodama $\cdot$ S. D. Hsieh $\cdot$ K. Kosaka $\cdot$ H. Sone $(\square)$

Health Management Center, Toranomon Hospital,

2-2-2 Toranomon,

Minato-ku, Tokyo 105-8470, Japan

e-mail: hsone@md.tsukuba.ac.jp

S. Hara $\cdot$ Y. Arase $\cdot$ H. Tsuji $\cdot$ S. D. Hsieh $\cdot$ K. Kosaka

Okinaka Memorial Institute for Medical Research,

Toranomon Hospital,

Tokyo, Japan
Results During a 5 year follow-up, 250 individuals developed type 2 diabetes. The lowest tertile of serum potassium (2.8$3.9 \mathrm{mmol} / \mathrm{l})$ was independently associated with the development of diabetes after adjustment for known predictors (HR 1.57 [95\% CI, 1.15-2.15]) compared with the highest tertile $(4.2-5.4 \mathrm{mmol} / \mathrm{l})$. Every $0.5 \mathrm{mmol} / \mathrm{l}$ lower increment in the baseline serum potassium level was associated with a $45 \%$ $(12-87 \%)$ increased risk of diabetes.

Conclusions/interpretation Mild to moderately low serum potassium levels, within the normal range and without frank hypokalaemia, could be predictive of type 2 diabetes in apparently healthy Japanese men.

Keywords Cohort studies · Risk factors · Serum potassium concentration - Type 2 diabetes mellitus
Abbreviations
FPG Fasting plasma glucose
TBK Total body potassium
eGFR Estimated glomerular filtration rate

\section{Introduction}

Evidence has suggested that hypokalaemia or decreased serum potassium induced by diuretics is related to impaired glucose tolerance and increased risk of diabetes in hypertensive individuals $[1,2]$. Low serum potassium approximates body potassium depletion, which is difficult to assess clinically [3]. In a small number of healthy individuals experimentally placed on a low-potassium diet, significant falls in serum potassium and total body potassium (TBK) were suggested to induce glucose intolerance associated with impaired insulin secretion [4, 
5], which is controlled by ATP-sensitive potassium channels [6]. A large cohort study showed an inverse association between dietary potassium intake and risk of type 2 diabetes [7]. More recently and concomitantly with the performance of our study, an inverse association of serum potassium and increased risk of type 2 diabetes independent of diuretic use was reported in white Americans and African-Americans [8]. However, until the present report, this issue was not studied in Asians. We examined whether low serum potassium was associated with an increased risk of type 2 diabetes in apparently healthy Japanese men without dietary restrictions.

\section{Methods}

The Toranomon Hospital Health Management Center Study (TOPICS) included apparently healthy Japanese government employees who underwent annual multiphasic health screening examinations. Each examination included biochemical tests and standard questionnaires on demographic characteristics, medical history and health-related habits. The study attempted to elucidate the incidence of and risk factors for various diseases among the Japanese population. Data were collected on 21,362 men and 8,222 women who had baseline examinations from 1997 to 2002. For our purpose, we analysed data on 5208 men who received four or more annual examinations during a 5 year follow-up. There were too few incident cases of diabetes among women for a meaningful analysis (27/1513 women after exclusions).

Diabetes was defined according to ADA criteria of fasting plasma glucose (FPG) $\geq 7.0 \mathrm{mmol} / \mathrm{l}$, self-reported cliniciandiagnosed diabetes or $\mathrm{HbA}_{1 \mathrm{c}}$ level $\geq 6.5 \%$ [9]. Reasons for exclusion were diabetes at the baseline examination $(n=278)$, taking antihypertensive medication $(n=453)$, renal dysfunction (serum creatinine level $>177 \mu \mathrm{mol} / \mathrm{l}, n=3$ ), liver dysfunction (under outpatient treatment, $n=116$ ) or missing data on baseline characteristics $(n=59)$. Data from 4,409 men aged 25-80 years were eligible for analysis.

Overnight fasting blood samples were immediately placed into Vacutainer tubes and spun in a cold centrifuge. Serum potassium concentration was measured by the ionselective electrode method (Hitachi 008, Tokyo, Japan). Individuals were categorised into tertiles of serum potassium distribution. For comparison, data were analysed in four groups according to equal intervals of $0.3 \mathrm{mmol} / 1$ serum potassium $(<3.7,3.7-3.9,4.0-4.2$ and $\geq 4.3 \mathrm{mmol} / \mathrm{l})$ based on our institute's normal range $(3.7-4.8 \mathrm{mmol} / \mathrm{l})$. The association of change in serum potassium during follow-up with risk of type 2 diabetes was also investigated.

Statistical analysis HRs and 95\% CIs were estimated by Cox regression for each serum potassium category using the highest tertile $(4.2-5.4 \mathrm{mmol} / \mathrm{l})$ as the reference or potassium as a continuous variable. Follow-up time was calculated from the first examination to the date of confirmed diabetes or last follow-up examination. Tests for linear trends across increasing categories of serum potassium concentrations treated categories as continuous variables in a model. Changes in serum potassium from baseline were calculated only when data for 5 years of follow-up were available $(2,594$ men and 176 incident cases). SPSS version 17.0 (SPSS, Chicago, IL, USA) was used for statistical analysis; $p<0.05$ indicated statistical significance.

Informed consent was obtained from all participants. The study protocol followed the Japanese Government's Ethical Guidelines Regarding Epidemiological Studies in accordance with the Declaration of Helsinki and was reviewed by the Institutional Review Board at Toranomon Hospital.

\section{Results}

During 5 years of follow-up (median 4.98 years, 21,094 person-years), we documented 250 incident cases of type 2 diabetes ( $32 \%$ by self-report; $68 \%$ by FPG and/or $\mathrm{HbA}_{1 \mathrm{c}}$ ). No correlation between serum potassium at baseline and BMI gain during follow-up was revealed $(p=0.928)$. Older age was associated with higher serum potassium. The association between serum potassium and glucose or $\mathrm{HbA}_{1 \mathrm{c}}$ was attenuated after adjustment for age or a stratified analysis (Table 1).

Lower tertiles compared with the highest tertile of serum potassium trended to be associated with a higher HR for incident type 2 diabetes, but without statistical significance in the age-adjusted model (Table 2). The HR in the lowest tertile $(2.8-3.9 \mathrm{mmol} / \mathrm{l})$ became statistically significant after adjustment for $\mathrm{HbA}_{1 \mathrm{c}}$, and the lowest tertile of serum potassium was associated with an increased risk independent of traditional predictors. A similar association was observed for categories of equal intervals of $0.3 \mathrm{mmol} / \mathrm{l}$ of serum potassium. Results for lower values $(<3.7 \mathrm{mmol} / \mathrm{l})$ within the first tertile $(2.8-3.9 \mathrm{mmol} / \mathrm{l})$ were associated with even greater risk. The association between serum potassium and risk of diabetes was fundamentally the same when $\mathrm{HbA}_{1 \mathrm{c}}$ values were not included among criteria for type 2 diabetes. Among the entire group (4,423 men) there were 230 incident cases of diabetes. Multivariate-adjusted HR for the lowest compared with the highest tertile was 1.74 (1.25-2.41).

Cox analysis with potassium as a continuous variable showed that every $0.5 \mathrm{mmol} / 1$ lower serum potassium level at baseline was associated with a $45 \%(12-87 \%)$ higher risk of type 2 diabetes in the entire group (multivariate $+\mathrm{HbA}_{1 \mathrm{c}}$ and FPG model). When individuals were stratified according to baseline prediabetic state, low potassium levels were signifi- 
Table 1 Baseline characteristics of the study participants by tertiles of serum potassium levels

\begin{tabular}{|c|c|c|c|c|}
\hline \multirow[t]{2}{*}{ Variable } & \multicolumn{3}{|c|}{ Serum potassium (mmol/1) } & \multirow[t]{2}{*}{$p$ value for trend } \\
\hline & Tertile 1 (2.8-3.9) & Tertile $2(4.0-4.1)$ & Tertile $3(4.2-5.4)$ & \\
\hline$n$ & 1,296 & 1,402 & 1,711 & \\
\hline Serum potassium $(\mathrm{mmol} / \mathrm{l})$ & $3.8 \pm 0.1$ & $4.1 \pm 0.1$ & $4.4 \pm 0.2$ & $<0.001$ \\
\hline Age (years) & $47.2 \pm 7.9$ & $48.1 \pm 8.2$ & $49.7 \pm 8.8$ & $<0.001$ \\
\hline BMI $\left(\mathrm{kg} / \mathrm{m}^{2}\right)$ & $23.1 \pm 2.6$ & $23.2 \pm 2.7$ & $23.1 \pm 2.6$ & 0.935 \\
\hline FPG $(\mathrm{mmol} / \mathrm{l})$ & $5.3 \pm 0.4$ & $5.3 \pm 0.5$ & $5.3 \pm 0.4$ & 0.017 \\
\hline $\mathrm{HbA}_{1 \mathrm{c}}(\%)^{\mathrm{a}}$ & $5.2 \pm 0.3$ & $5.3 \pm 0.3$ & $5.3 \pm 0.4$ & $<0.001$ \\
\hline Systolic blood pressure $(\mathrm{mmHg})$ & $127 \pm 15$ & $124 \pm 14$ & $124 \pm 14$ & $<0.001$ \\
\hline Diastolic blood pressure $(\mathrm{mmHg})$ & $77 \pm 11$ & $76 \pm 10$ & $76 \pm 10$ & 0.162 \\
\hline Prevalence of hypertension $(\%)^{\mathrm{b}}$ & 18.6 & 14.1 & 14.6 & 0.002 \\
\hline Total cholesterol (mmol/l) & $5.17 \pm 0.82$ & $5.22 \pm 0.83$ & $5.27 \pm 0.83$ & 0.001 \\
\hline HDL-cholesterol (mmol/l) & $1.31 \pm 0.36$ & $1.30 \pm 0.35$ & $1.31 \pm 0.36$ & 1.000 \\
\hline Triacylglycerol (mmol/l) & $1.21(0.85-1.73)$ & $1.15(0.85-1.67)$ & $1.19(0.86-1.75)$ & 0.633 \\
\hline Uric acid $(\mu \mathrm{mol} / 1)$ & $355.4 \pm 70.5$ & $361.9 \pm 74.3$ & $365.3 \pm 72.0$ & $<0.001$ \\
\hline eGFR $\left(\mathrm{ml} \mathrm{min} \operatorname{mon}^{-1} 1.73 \mathrm{~m}^{-2}\right)^{\mathrm{c}}$ & $76.4 \pm 12.2$ & $75.8 \pm 11.9$ & $74.1 \pm 12.4$ & $<0.001$ \\
\hline$<60 \mathrm{ml} \mathrm{min}{ }^{-1} 1.73 \mathrm{~m}^{-2}(\%)$ & 7.1 & 8.4 & 11.9 & $<0.001$ \\
\hline Smoking (never/former/current) (\%) & $47.4 / 28.5 / 24.1$ & $42.2 / 29.0 / 28.7$ & $34.8 / 30.4 / 34.8$ & $<0.001$ \\
\hline Parental diabetes $(\%)$ & 13.7 & 13.1 & 13.3 & 0.894 \\
\hline
\end{tabular}

Data are $n(\%)$, mean $\pm \mathrm{SD}, \%$ or median (interquartile ranges) unless otherwise indicated

${ }^{a} \mathrm{HbA}_{1 \mathrm{c}}$ determined as the National Glycohemoglobin Standardization Program: value $(\%)=0.0981 \times(10.39 \times[$ Japan Diabetes Society Committee for the Standardization value (\%)]-16.8) +1.95

${ }^{\mathrm{b}}$ Hypertension defined as systolic blood pressure $\geq 140 \mathrm{mmHg}$ or diastolic blood pressure $\geq 90 \mathrm{mmHg}$

${ }^{\mathrm{c}} \mathrm{eGFR}=194 \times$ creatinine $^{-1.094} \times \mathrm{age}^{-0.287}$

$p$ values for trend were tested by ANOVA for continuous variables and $\chi^{2}$ test for categorical variables

eGFR, estimated glomerular filtration rate

cantly associated with an increased risk of type 2 diabetes, particularly among prediabetic individuals at baseline.

During follow-up, 315 individuals began antihypertensive therapy, but results differed little from those not taking antihypertensives. There was a 50\% (15-96\%) adjusted increased risk for each $0.5 \mathrm{mmol} / 1$ lower increment of serum potassium at baseline.

Quintile distribution of serum potassium changes suggested that larger decreases in serum potassium after baseline were associated with increasingly higher incident rates regardless of baseline serum potassium (quintile [Q] 1 less than $-0.1 \mathrm{mmol} / 1,9.0 \%$; Q2 $-0.1-0.0 \mathrm{mmol} / 1,8.6 \%$; Q3 $0.1 \mathrm{mmol} / 1,6.7 \%$; Q4 $0.2 \mathrm{mmol} / 1,5.6 \%$; and Q5 $\geq 0.3 \mathrm{mmol} / 1,4.7 \% ; p=0.001$ for trend).

\section{Discussion}

This prospective study showed an association between lower serum potassium and increased risk of type 2 diabetes in Japanese men not using antihypertensive medications. Also, the results suggested hypokalaemia as a possible factor in progression from a prediabetic state to type 2 diabetes.

Our results coincide with studies of both hypertensive and healthy individuals $[1,2,4,5,8]$. Among hypertensive patients, every $0.5 \mathrm{mmol} / 1$ decrease induced by diuretics was associated with a $45 \%$ higher risk of diabetes [2]. Maintaining serum potassium above $4.0 \mathrm{mmol} / \mathrm{l}$ was reported to be useful in preventing thiazide-induced diabetes [1].

Glucose intolerance associated with decreased insulin secretion was induced in healthy individuals placed in a hypokalaemic state (2.4-3.6 [4] or 2.4-3.5 $\mathrm{mmol} / \mathrm{l}$ by dietary restrictions [5]). The Atherosclerosis Risk in Communities (ARIC) study of white Americans and African-Americans in the USA [8] reported an independent association of low-normal serum potassium $(2.5-5.0 \mathrm{mmol} / \mathrm{l})$ with development of type 2 diabetes compared with highnormal levels (5.0-5.5 mmol/l), while our results showed an independent association of values $<4.0 \mathrm{mmol} / \mathrm{l}$ with an increased risk of type 2 diabetes. Our results for an Asian study population agree with the results from the US study that a mild-to-moderate decrease in serum potassium, even within 


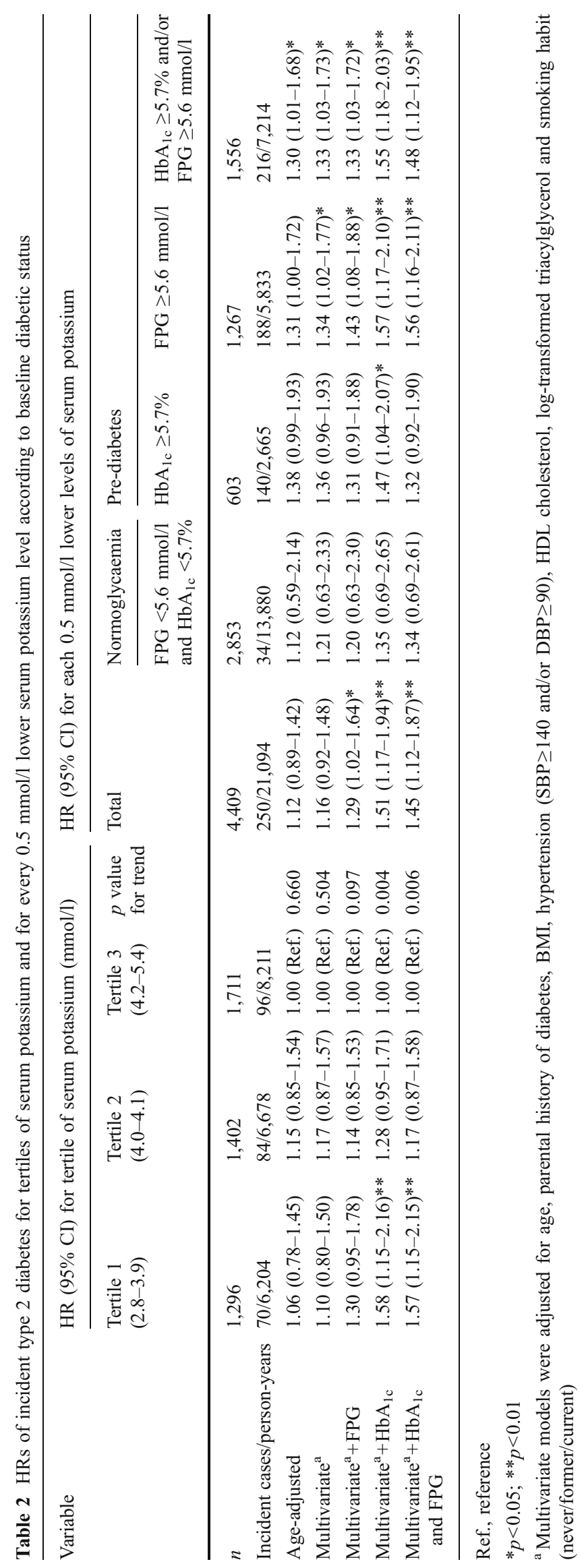


normal range and without frank hypokalaemia, could influence the development of type 2 diabetes. Lower serum potassium appears important in the risk of type 2 diabetes across various ethnic groups.

Being normoglycaemic, the majority of our study participants were assumed to have normal insulin secretion at baseline. However, that early-phase insulin decreased progressively when FPG exceeded $5.6 \mathrm{mmol} / \mathrm{l}$ indicates an important role for early-phase insulin secretion in the development of glucose intolerance, specifically in Asians [10]. Therefore, hypokalaemia could influence the development of type 2 diabetes among prediabetic individuals, that is, those having metabolic abnormalities of reduced TBK and diminished insulin secretion. In addition, as $\mathrm{HbA}_{1 \mathrm{c}}$ reflects chronic hyperglycaemia rather than acute dysglycaemia, robust results might be shown after adjustment for $\mathrm{HbA}_{1 \mathrm{c}}$.

Low dietary potassium intake of $40 \mathrm{mmol} /$ day resulted in a significant fall in serum potassium and TBK, leading to glucose intolerance in normal individuals [4]. Theoretically, a $0.3 \mathrm{mmol} / 1$ decrease in serum potassium corresponds to a 100 mmol reduction in TBK on average [3]. However, because redistribution between extracellular and intracellular spaces also influences serum potassium, hypokalaemia should be considered to coexist with normal body potassium levels. As TBK values were unavailable, we could not address the role of TBK. However, we found that in Japanese men without dietary restrictions, serum potassium, a widely available measurement in clinical practice, was associated with incident type 2 diabetes.

The study strengths were the large sample size, strict exclusion criteria, especially excluding those taking diuretics at baseline, and the availability of $\mathrm{HbA}_{1 \mathrm{c}}$ data. The limitations were that all participants were men, neither serum magnesium nor insulin was measured and information on potassium supplementation or dietary intake was unavailable. Whether poor dietary potassium intake affected serum potassium and subsequently influenced our results could not be determined, although no independent association between dietary potassium intake and risk of diabetes has been shown [8]. Factors such as lifestyle might have influenced results. That managing serum potassium is effective in preventing diabetes cannot be confirmed from the present results. Further research is required. Also, the association between changes in serum potassium and risk of diabetes in healthy individuals should be studied.

In conclusion, low serum potassium even within the normal range could be predictive of type 2 diabetes in healthy Japanese men not using antihypertensive medications. This association might be universal, regardless of ethnicity.

Acknowledgements This work is supported in part by the Ministry of Health, Labour and Welfare, Japan. H. Sone and S. Kodama are recipients of a Grant-in-Aid for Scientific Research and Postdoctoral Research Fellowship, respectively, both from the Japan Society for the Promotion of Science. The sponsor had no role in the design and conduct of the study.

Duality of interest The authors declare that there is no duality of interest associated with this manuscript.

\section{References}

1. Zillich AJ, Garg J, Basu S, Bakris GL, Carter BL (2006) Thiazide diuretics, potassium, and the development of diabetes: a quantitative review. Hypertension 48:219-224

2. Shafi T, Appel LJ, Miller ER 3rd, Klag MJ, Parekh RS (2008) Changes in serum potassium mediate thiazide-induced diabetes. Hypertension 52:1022-1029

3. Sterns RH, Cox M, Feig PU, Singer I (1981) Internal potassium balance and the control of the plasma potassium concentration. Medicine (Baltimore) 60:339-354

4. Rowe JW, Tobin JD, Rosa RM, Andres R (1980) Effect of experimental potassium deficiency on glucose and insulin metabolism. Metabolism 29:498-502

5. Gorden P (1973) Glucose intolerance with hypokalemia. Failure of short-term potassium depletion in normal subjects to reproduce the glucose and insulin abnormalities of clinical hypokalemia. Diabetes 22:544-551

6. Sperling MA (2006) ATP-sensitive potassium channels - neonatal diabetes mellitus and beyond. N Engl J Med 355:507-510

7. Colditz GA, Manson JE, Stampfer MJ, Rosner B, Willett WC, Speizer FE (1992) Diet and risk of clinical diabetes in women. Am J Clin Nutr 55:1018-1023

8. Chatterjee R, Yeh HC, Shafi T et al (2010) Serum and dietary potassium and risk of incident type 2 diabetes mellitus: the Atherosclerosis Risk in Communities (ARIC) Study. Arch Intern Med 170:1745-1751

9. American Diabetes Association (2010) Standards of medical care in diabetes-2010. Diabetes Care 33(Suppl 1):S11-S61

10. Matsumoto K, Miyake S, Yano M et al (1997) Glucose tolerance, insulin secretion, and insulin sensitivity in nonobese and obese Japanese subjects. Diabetes Care 20:1562-1568 